\title{
Forward Prediction in the Posterior Parietal Cortex and Dynamic Brain-Machine Interface
}

\author{
$\mathrm{He} \mathrm{Cui}^{1,2 *}$ \\ ${ }^{1}$ Institute of Neuroscience, Chinese Academy of Sciences (CAS), Shanghai, China, ${ }^{2}$ Key Laboratory of Primate \\ Neurobiology, Chinese Academy of Sciences (CAS), Shanghai, China
}

While remarkable progress has been made in brain-machine interfaces (BMls) over the past two decades, it is still difficult to utilize neural signals to drive artificial actuators to produce predictive movements in response to dynamic stimuli. In contrast to naturalistic limb movements largely based on forward planning, brain-controlled neuroprosthetics mainly rely on feedback without prior trajectory formation. As an important sensorimotor interface integrating multisensory inputs and efference copy, the posterior parietal cortex (PPC) might play a proactive role in predictive motor control. Here it is proposed that predictive neural activity in PPC could be decoded to provide prosthetic control signals for guiding BMl systems in dynamic environments.

Keywords: neuroprosthetics, decoding, neuroengineering, internal model, motor control, paralysis

OPEN ACCESS

Edited by:

Henry H. Yin,

Duke University, USA

Reviewed by:

Hugo Merchant,

National Autonomous University of

Mexico, Mexico

Xin Jin,

Salk Institute for Biological

Studies, USA

*Correspondence:

$\mathrm{He} \mathrm{Cui}$

cuihe@ion.ac.cn

Received: 17 August 2016 Accepted: 10 October 2016 Published: 26 October 2016

Citation:

Cui H (2016) Forward Prediction in the Posterior Parietal Cortex and

Dynamic Brain-Machine Interface.

Front. Integr. Neurosci. 10:35.

doi: 10.3389/fnint.2016.00035

\section{INTRODUCTION}

To interact with a changing world, such as in tracking and intercepting moving objects, the brain must overcome pervasive sensorimotor delays (Nijhawan, 2008; Franklin and Wolpert, 2011). Although it has been proposed that compensating for these inherent delays is based on bottom-up sensory extrapolation (e.g., the flash-lag effect, Nijhawan, 1994; Nijhawan and Wu, 2009), the prevalent view of sensorimotor control posits that action planning relies on forward models based on an intimate interplay between sensory inflow and motor outflow, rather than a hierarchical transformation from extrinsic stimuli to intrinsic muscular activity (Wolpert et al., 1995; Shadmehr and Mussa-Ivaldi, 2012).

\section{FORWARD MODEL FOR PREDICTIVE SENSORIMOTOR CONTROL}

Movement planning predominately arises from an internal prediction of future states of body and environment, instead of merely relying on sensory responses. Since the emergence of the concept of forward models, important advances have been made in understanding how efference copies of motor commands are routed back to sensory structures for internally monitoring movement (Sommer and Wurtz, 2008). Those signals, widely referred as corollary discharges, have been observed across different species at many levels, including the cerebral cortex, spinal cord, cerebellum and muscle spindles (Crapse and Sommer, 2008). Through such 
wide-spread feedback in the form of closed sensorimotor loops, the brain is able to distinguish external motion from self-generated movements (Angelaki and Cullen, 2008), update sensory representations (Duhamel et al., 1992) and motor execution (Azim et al., 2014), and optimize active sensation (Kleinfeld and Deschênes, 2011). However, it is unclear where and how re-afferent signals are integrated with sensory inputs to form forward predictions leading to future movements, rather than solely monitoring them.

Most studies in sensorimotor neurophysiology have utilized reactive movements to stationary goals pre-defined by sensory cues (Figure 1A left), but this approach is fundamentally incapable of determining whether the observed neural activity reflects sensory stimuli or predicts future states. Exploring the neural codes of a forward model demands the development of novel behavioral tasks that are highly dependent on predictive spatiotemporal transformations, such as interception (Figure 1A right), in which the movement is directed to a predicted future location of a moving target, as opposed to a static location explicitly specified by sensory cues. Interception has been widely investigated in numerous studies (see reviews Merchant and Georgopoulos, 2006; Zago et al., 2009). In the temporal domain, Merchant et al. $(2004 a, b)$ have shown that activity in both parietal and motor cortices encode estimations of arrival time (Tau-coupling, Lee, 1976) for precisely-timed interception at pre-determined destinations. In contrast to a wealth of data on temporal prediction, spatial extrapolation for interception has rarely been addressed, and consequentially little is known about its neural implementation.

During tracking and pursuing of moving stimuli, neuronal activity faithfully conveying the instantaneous or inferred target motion has been found in both cortical (e.g., Assad and Maunsell, 1995; Ferrera and Barborica, 2010) and subcortical areas (e.g., Cui and Malpeli, 2003; Ma et al., 2013). However, the neurons examined in these studies were primarily involved in the formation of upto-date percepts, rather than in specifying the goals of the intercepting movements. For example, during catch-up saccades toward moving targets, pre-saccadic activity in the superior colliculus (SC) encodes retinal position error, instead of actual saccade metrics that take into account retinal slip (Keller et al., 1996). It is unclear how the time involved in actual generation and completion of the eye movements is taken into account.

\section{PLAUSIBLE ROLE OF THE PPC IN FORWARD PREDICTION}

Internal prediction of the sensory consequences of extra-personal objects is, presumably, a high-order sensory representation that might be embodied in association areas. Moreover, forward prediction demands integrating different sources of sensorimotor information through a rich pattern of anatomical connectivity. As a crucial node incorporating visual, proprioceptive and efference copy information in a sensorimotor network (Andersen et al., 1997), the

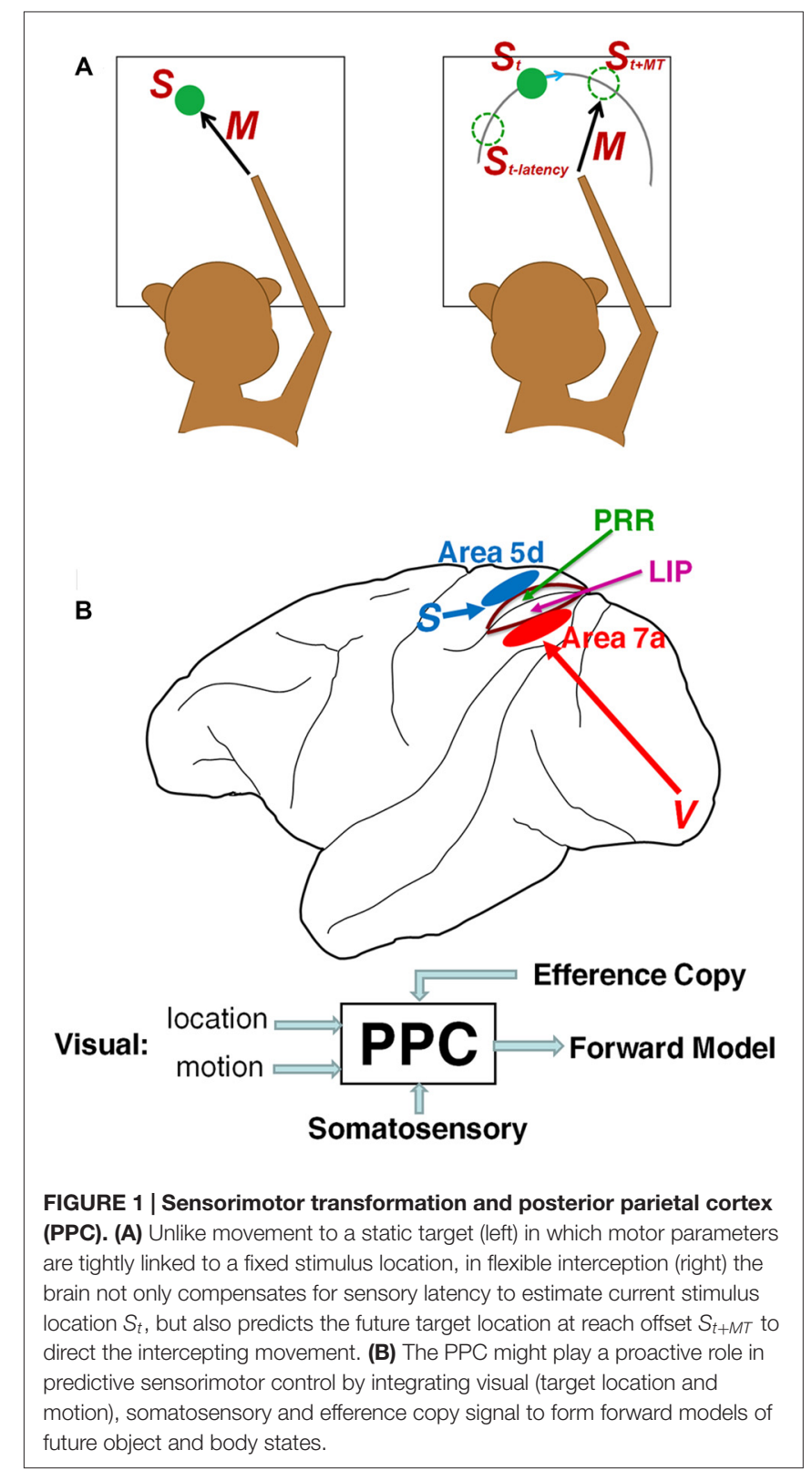

posterior parietal cortex (PPC) is a plausible candidate for mediating the fundamental relationship between sensory prediction and motor control (Figure 1B). Examining PPC activity during interceptive behaviors in dynamic environments might provide deep insights into how the brain constructs forward predictions for guiding movement, because the internal prediction of future target location can be inferred from the intercepting movements themselves. If the PPC underlies the forward model, PPC neurons should not merely convey the current states of body and object, but also should predict the future consequences of an impending movement. Indeed, accumulating evidence indicates that PPC activity predicts the sensory consequences of upcoming movements (Mulliken and Andersen, 2009). Thus, the PPC might fulfill the goal of forward prediction 
in sensorimotor transformations, and work in concert with inverse models in the motor cortex and the subcortical motor apparatus to implement them (Andersen and Cui, 2009).

Prediction is not only fundamental for motor control, but may also be crucial for many aspects of cognition, including sequential planning, decision making, social interaction, action understanding, imitation and mental practice. Therefore, forward prediction might offer a cohesive framework for understanding the neural basis of many related behaviors. For instance, neurological studies have shown that patients with lesion in the left PPC suffer from ideational apraxia (Buxbaum, 1998; Zadikoff and Lang, 2005). Although these patients appear normal for simple movements, they are impaired in associating objects with their purposes, and/or in generating complicated action sequences. A plausible interpretation is that the PPC damage interfered with the prediction of outcomes and consequences of forthcoming actions.

Growing evidence suggests that the PPC is composed of multiple functional subareas with distinct roles in the sensorimotor transformation (Andersen and Buneo, 2002; Cui, 2014). These include inferior parietal area $7 \mathrm{a}$ and superior parietal area $5 \mathrm{~d}$, as well as their upstream structures, and also the lateral intraparietal area (LIP) and parietal reach region (PRR; Figure 1B). Area $7 \mathrm{a}$ is the top structure in a dorsal visual hierarchy (Felleman and Van Essen, 1991; Bastos et al., 2014), with rich inputs from motion-sensitive extrastriate areas (Felleman and Van Essen, 1991). Its activity is modulated by world-centered gain fields (Snyder et al., 1998) and other top-down inputs from the prefrontal cortex (Crowe et al., 2013), hippocampus and cerebellum (Clower et al., 2001). Consequently, it is ideally positioned to represent the visual prediction of behaviorally relevant objects achieved through sensorimotor learning.

As a counterpart of area $7 \mathrm{a}$, area 5 initially was thought of as a high-level somatosensory area (even sometimes referred to as S3) that conveys more abstract information about combined joint angles (Sakata et al., 1973). However, subsequent studies on behaving monkeys have shown that area 5 neurons convey kinematic information (Ashe and Georgopoulos, 1994; Kalaska and Crammond, 1995) for component arm movements ( $\mathrm{Li}$ and Cui, 2013) in body-related coordinates (Johnson et al., 1996; Bremner and Andersen, 2012) by integrating visual and somatosensory inputs according to the behavioral context (Brunamonti et al., 2016). Given its close linkages to $\mathrm{S} 1$ and M1/PMd, area 5 might predict the somatosensory consequence of an upcoming arm movement during interception, with its neural activity primarily associated with intrinsic kinematic characteristics, such as limb trajectories, speed profiles and joint angles.

To investigate neural activity when movements are directed by anticipated sensory outcomes, rather than by current perceived stimulus locations, we have recorded parietal activity from monkeys performing a flexible manual interception

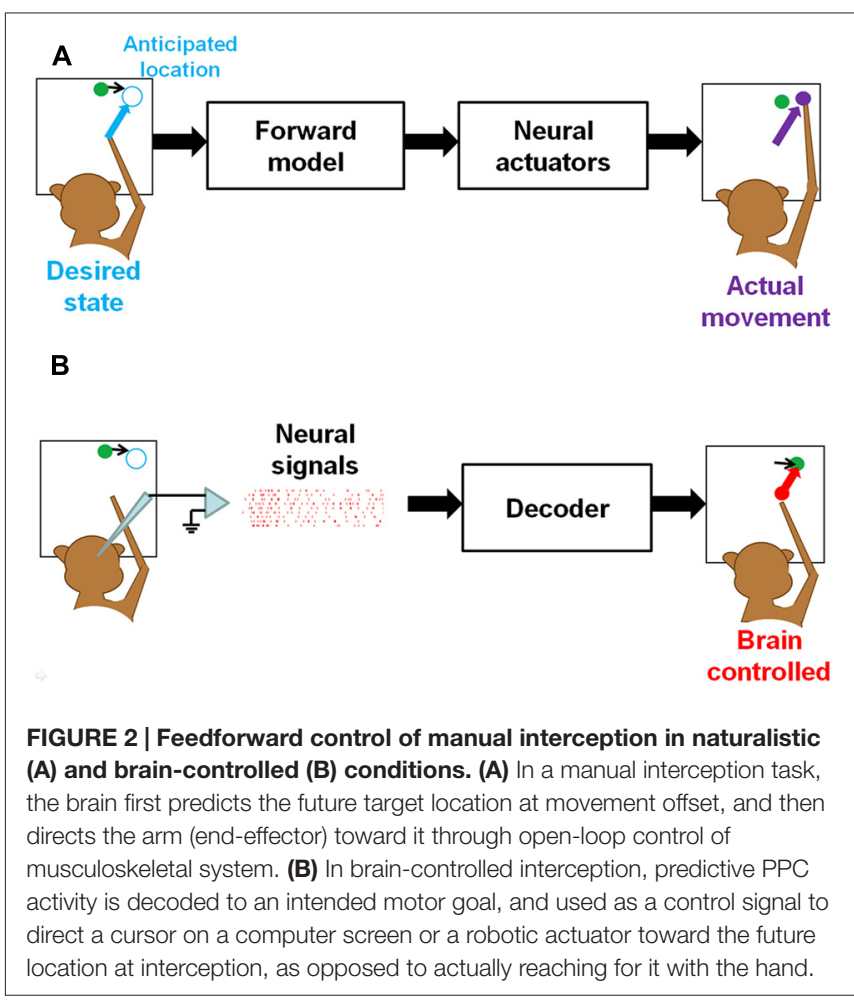

task involving dynamic sensory-motor contingencies. In this paradigm, the monkey initiates a trial by positioning a hand at the center of a touch screen. A peripheral target moving at an angular velocity of 0 (control), 120, or $240^{\circ} / \mathrm{s}$ in a circular path appears in one of eight locations spaced at $45^{\circ}$. The targets, which could be moving either clockwise or counter clockwise, is visible for $1 \mathrm{~s}$, have to be intercepted by a hand movement within this interval. During interception, a hand movement should be planned toward the anticipated target location at interception to accomplish the task. Therefore, if area $7 \mathrm{a}$ predicts the visual consequences of the upcoming interception, its pre-movement activity should encode movement destination, rather than instantaneous stimulus location. Preliminary data support this idea (Li et al., 2014). They suggest that movement-directional tuning curves of pre-movement activity are invariant, but stimulus-directional tuning curves significantly shift as functions of target speed. Partial correlation analysis demonstrates that PPC activity is more pronounced for the reaching direction than for the stimulus location, suggesting an intimate role in forward prediction and motor planning.

\section{DECODING PPC ACTIVITY FOR FEEDFORWARD PROSTHETIC CONTROL}

Over the past two decades, intracortically-based neuroprosthetics have emerged as promising approaches to restoring sensorimotor function for severely disabled patients suffering from nervous diseases or injuries. Based on neural activity recorded from chronically implanted multi-channel electrode arrays, 
intended movements have been successfully decoded as command signals and used to manipulate a robotic device or a cursor on a computer screen to replace the lost motor function of a paralyzed limb (Serruya et al., 2002; Carmena et al., 2003; Musallam et al., 2004; Hochberg et al., 2006; Santhanam et al., 2006; Velliste et al., 2008; Aflalo et al., 2015), even enabling it bimanually (Ifft et al., 2013) or bi-directionally (Ethier et al., 2012). Although such brain-machine interfaces (BMIs) have succeeded in continuously driving prosthetic arms, the achieved performances in movement speed, straightness and smoothness still fall short of widespread clinical applicability. Unlike natural movements planned in a feedforward manner, brain-controlled prosthetic devices demand continuous guidance of decoded neural signals, largely relying on visual feedback during movement execution. Furthermore, in a dynamic world, it is unlikely that closed-loop prosthetic systems depending on sensory feedback control are feasible for capturing moving objects.

Clinically viable BMI systems that enable real-time interactions with dynamic environments demand translation of predictive neural activity into desired motor goals to guide ballistic movements, as in natural interception (Figure 2A). Since our ongoing research has indicated that pre-movement activity in the PPC is informative of the intercepting movement, it is, in principle, possible to decoded this activity and utilize it as a predictor of an upcoming movement destination. Based on the decoded endpoint position, a computer cursor or artificial limb could be moved by a goal-directed open-loop controller (Figure 2B). After the desired endpoint is extracted from the PPC activity by an optimal estimation decoder (i.e., population vector, machine learning algorithm), the artificial actuator will be driven toward the intended movement goal while receiving no further brain controls until landing. If the actuator is a computer cursor, it will move to the goal in a bell-shaped speed profile, as described by Fitts' law. If the actuator is a high-degree of freedom (DOF) robotic limb, it will be driven by a goal-directed pre-coded program fitted to its own mechanics, rather than by biomimetic kinematics. Of course, how the central nervous system compensates for prosthetic motor errors through learning and adaptation to archieve proficient BMI control is a challenging problem.

\section{REFERENCES}

Aflalo, T., Kellis, S., Klaes, C., Lee, B., Shi, Y., Pejsa, K., et al. (2015). Decoding motor imagery from the posterior parietal cortex of a tetraplegic human. Science 348, 906-910. doi: 10.1126/science.aaa5417

Andersen, R. A., and Buneo, C. A. (2002). Intentional maps in posterior parietal cortex. Annu. Rev. Neurosci. 25, 189-220. doi: 10.1146/annurev.neuro.25. 112701.142922

Andersen, R. A., and Cui, H. (2009). Intention, action planning and decision making in parietal-frontal circuits. Neuron 63, 568-583. doi: 10.1016/j.neuron. 2009.08.028

Andersen, R. A., Snyder, L. H., Bradley, D. C., and Xing, J. (1997). Multimodal representation of space in the posterior parietal cortex and its use in planning

\section{DISCUSSION}

Even if predictive activity embodied in the PPC can provide useful neural signals for open-loop prosthetic control of movements, important issues remained to be addressed. Predictive sensorimotor control engages a highly distributed cortical network, so the PPC alone probably is inadequate for directing comprehensive and adaptable motor repertoires. Preferably, a PPC-based controller should be integrated with neural signals from motor areas to implement timely and accurate control of rapidly-moving actuators. Furthermore, because successful interception requires not only accurate spatial prediction, but also precise timing (Merchant and Georgopoulos, 2006; Zago et al., 2009), dynamic BMIs based on the PPC should also incorporate temporal information conveyed in the motor and premotor cortex (Merchant and Georgopoulos, 2006; Merchant et al., 2009, 2011).

Despite the importance of forward models, movement can still be modified by sensory feedback during execution (Todorov and Jordan, 2002; Scott, 2004). According to the behavioral context, natural interceptions might adopt reactive, predictive, or mixed strategies (Port et al., 1997; Merchant et al., 2003). Thus, prosthetic movements directed to an endpoint decoded from PPC signals could be corrected online by an artificial proprioceptive feedback via intracortical microstimulation (Dadarlat et al., 2015), probably in the deceleration phase.

In summary, BMIs capable of implementing predictive sensorimotor control in dynamic circumstances require systematic integration of modern motor neuroscience and numerous sophisticated techniques. If successful, such an approach may allow paralyzed patients to bypass their immobilized bodies, and interact with the dynamic external world.

\section{AUTHOR CONTRIBUTIONS}

HC conceived the study and wrote the article.

\section{ACKNOWLEDGMENTS}

The author thanks J.G. Malpeli for helpful edits on the manuscript, and P. Ding, S. Guo, C. Li, M. Wang, T. Wang and Y. Zhang for useful discussions.

movements. Annu. Rev. Neurosci. 20, 303-330. doi: 10.1146/annurev.neuro.20. 1.303

Angelaki, D. E., and Cullen, K. E. (2008). Vestibular system: the many facets of a multimodal sense. Annu. Rev. Neurosci. 31, 125-150. doi: 10.1146/annurev. neuro.31.060407.125555

Ashe, J., and Georgopoulos, A. P. (1994). Movement parameters and neural activity in motor cortex and area 5. Cereb. Cortex 4, 590-600. doi: 10 1093/cercor/4.6.590

Assad, J. A., and Maunsell, J. H. (1995). Neuronal correlates of inferred motion in primate posterior parietal cortex. Nature 373, 518-521. doi: 10.1038/373518a0

Azim, E., Jiang, J., Alstermark, B., and Jessell, T. M. (2014). Skilled reaching relies on a V2a propriospinal internal copy circuit. Nature 508, 357-363. doi: 10. 1038/nature13021 
Bastos, A. M., Vezoli, J., Bosman, C. A., Schoffelen, J. M., Oostenveld, R., and Dowdall, J. R. (2014). Visual areas exert feedforward and feedback influences through distinct frequency channels. Neuron 85, 390-401. doi: 10.1016/j. neuron.2014.12.018

Bremner, L. R., and Andersen, R. A. (2012). Coding of the reach vector in parietal area 5d. Neuron 75, 342-351. doi: 10.1016/j.neuron.2012.03.041

Brunamonti, E., Genovesio, A., Pani, P., Caminiti, R., and Ferraina, S. (2016). Reaching-related neurons in superior parietal area 5: influence of the target visibility. J. Cogn. Neurosci. 28, 1828-1837. doi: 10.1162/jocn_a_01004

Buxbaum, L. J. (1998). Ideational apraxia and naturalistic action. Cogn. Neuropsychol. 15, 617-643. doi: 10.1080/026432998381032

Carmena, J. M., Lebedev, M. A., Crist, R. E., O’Doherty, J. E., Santucci, D. M., Dimitrov, D. F., et al. (2003). Learning to control a brain-machine interface for reaching and grasping by primates. PLoS Biol. 1:e42. doi: 10.1371/journal.pbio. 0000042

Clower, D. M., West, R. A., Lynch, J. C., and Strick, P. L. (2001). The inferior parietal lobule is the target of output from the superior colliculus, hippocampus and cerebellum. J. Neurosci. 21, 6283-6291.

Crapse, T. B., and Sommer, M. A. (2008). Corollary discharge across the animal kingdom. Nat. Rev. Neurosci. 9, 587-600. doi: 10.1038/nrn2457

Crowe, D. A., Goodwin, S. J., Blackman, R. K., Sakellaridi, S., Sponheim, S. R., and MacDonald, A. W. III (2013). Prefrontal neurons transmit signals to parietal neurons that reflect executive control of cognition. Nat. Neurosci. 16, 1484-1491. doi: 10.1038/nn.3509

Cui, H. (2014). From intention to action: hierarchical sensorimotor transformation in the posterior parietal cortex. eNeuro 1:e0017-14.2014. doi: 10.1523/ENEURO.0017-14.2014

Cui, H., and Malpeli, J. G. (2003). Activity in the parabigeminal nucleus during eye movements directed at moving and stationary targets. J. Neurophysiol. 89, 3128-3142. doi: 10.1152/jn.01067.2002

Dadarlat, M. C., O’Doherty, J. E., and Sabes, P. N. (2015). A learning-based approach to artificial sensory feedback leads to optimal integration. Nat. Neurosci. 18, 138-144. doi: 10.1038/nn.3883

Duhamel, J. R., Colby, C. L., and Goldberg, M. E. (1992). The updating of the representation of visual space in parietal cortex by intended eye movements. Science 55, 90-92. doi: 10.1126/science.1553535

Ethier, C., Oby, E. R., Bauman, M. J., and Miller, L. E. (2012). Restoration of grasp following paralysis through brain-controlled stimulation of muscles. Nature 485, 368-371. doi: 10.1038/nature 10987

Felleman, D. J., and Van Essen, D. C. (1991). Distributed hierarchical processing in the primate cerebral cortex. Cereb. Cortex 1, 1-47. doi: 10.1093/cercor/1.1.1

Ferrera, V. P., and Barborica, A. (2010). Internally generated error signals in monkey frontal eye field during an inferred motion task. J. Neurosci. 30, 11612-11623. doi: 10.1523/JNEUROSCI.2977-10.2010

Franklin, D. W., and Wolpert, D. M. (2011). Computational mechanisms of sensorimotor control. Neuron 72, 425-442. doi: 10.1016/j.neuron.2011.10.006

Hochberg, L. R., Serruya, M. D., Friehs, G. M., Mukand, J. A., Saleh, M., Caplan, A. H., et al. (2006). Neuronal ensemble control of prosthetic devices by a human with tetraplegia. Nature 442, 164-171. doi: 10.1038/nature04970

Ifft, P. J., Shokur, S., Li, Z., Lebedev, M. A., and Nicolelis, M. A. L. (2013). A brainmachine interface enables bimanual arm movements in monkeys. Sci. Transl. Med. 5:210ra154. doi: 10.1126/scitranslmed.3006159

Johnson, P. B., Ferraina, S., Bianchi, L., and Caminiti, R. (1996). Cortical networks for visual reaching: physiological and anatomical organization of frontal and parietal lobe arm regions. Cereb. Cortex 6, 102-119. doi: 10.1093/cercor/6.2. 102

Kalaska, J. F., and Crammond, D. J. (1995). Deciding not to GO: neuronal correlates of response selection in a GO/NOGO task in primate premotor and parietal cortex. Cereb. Cortex 5, 410-428. doi: 10.1093/cercor/5.5.410

Keller, E. L., Gandhi, N. J., and Weir, P. T. (1996). Discharge of superior collicular neurons during saccades made to moving targets. J. Neurophysiol. 76, 3573-3577.

Kleinfeld, D., and Deschênes, M. (2011). Neuronal basis for object location in the vibrissa scanning sensorimotor system. Neuron 72, 455-468. doi: 10.1016/j. neuron.2011.10.009

Lee, D. N. (1976). A theory of visual control of braking based on information about time-to-collision. Perception 5, 437-459. doi: 10.1068/p0 50437
Li, Y., and Cui, H. (2013). Dorsal parietal area 5 encodes immediate reach in sequential arm movements. J. Neurosci. 33, 14455-14465. doi: 10. 1523/JNEUROSCI.1162-13.2013

Li, Y., Wang, Y., and Cui, H. (2014). Posterior parietal cortex predicts upcoming movement during manual interception of moving targets. Society for Neuroscience Abstract, 437.03 .

Ma, R., Cui, H., Lee, S. H., Anastasio, T. J., and Malpeli, J. G. (2013). Predictive encoding of moving target trajectory by neurons in the parabigeminal nucleus. J. Neurophysiol. 109, 2029-2043. doi: 10.1152/jn. 01032.2012

Merchant, H., Battaglia-Mayer, A., and Georgopoulos, A. P. (2003). Interception of real and apparent motion targets: psychophysics in humans and monkeys. Exp. Brain Res. 152, 106-112. doi: 10.1007/s00221-0031514-5

Merchant, H., Battaglia-Mayer, A., and Georgopoulos, A. P. (2004a). Neural responses during interception of real and apparent circularly moving stimuli in motor cortex and area 7a. Cereb. Cortex 14, 314-331. doi: 10. 1093/cercor/bhg130

Merchant, H., Battaglia-Mayer, A., and Georgopoulos, A. P. (2004b). Neural responses in motor cortex and area 7a to real and apparent motion. Exp. Brain Res. 154, 291-307. doi: 10.1007/s00221-003-1664-5

Merchant, H., and Georgopoulos, A. P. (2006). Neurophysiology of perceptual and motor aspects of interception. J. Neurophysiol. 95, 1-13. doi: 10.1152/jn.00422. 2005

Merchant, H., Zarco, W., Pérez, O., Prado, L., and Bartolo, R. (2011). Measuring time with different neural chronometers during a synchronization-continuation task. Proc. Natl. Acad. Sci. U S A 108, 19784-19789. doi: 10.1073/pnas.1112933108

Merchant, H., Zarco, W., Prado, L., and Pérez, O. (2009). Behavioral and neurophysiological aspects of target. Adv. Exp. Med. Biol. 629, 201-220. doi: 10 1007/978-0-387-77064-2_10

Mulliken, G. H., and Andersen, R. A. (2009). "Forward models and state estimation in posterior parietal cortex," in The Cognitive Neurosciences $I V$, ed. M. S. Gazzaniga (Cambridge, MA: MIT Press), 599-611.

Musallam, S., Corneil, B. D., Greger, B., Scherberger, H., and Andersen, R. A. (2004). Cognitive control signals for neural prosthetics. Science 305, 258-262. doi: $10.1126 /$ science. 1097938

Nijhawan, R. (1994). Motion extrapolation in catching. Nature 370, 256-257. doi: $10.1038 / 370256 \mathrm{~b} 0$

Nijhawan, R. (2008). Visual prediction: psychophysics and neurophysiology of compensation for time delays. Behav. Brain Sci. 31, 179-198; discussion 198-239. doi: 10.1017/S0140525X08003804

Nijhawan, R., and Wu, S. (2009). Compensating time delays with neural predictions: are predictions sensory or motor? Philos. Trans. A Math. Phys. Eng. Sci. 367, 1063-1078. doi: 10.1098/rsta.2008.0270

Port, N., Lee, D., Dassonville, P., and Georgopoulos, A. P. (1997). Manual interception of moving targets I. Performance and movement initiation. Exp. Brain Res. 116, 406-420. doi: 10.1007/pl00005769

Sakata, H., Takaoka, Y., Kawarasaki, A., and Shibutani, H. (1973). Somatosensory properties of neurons in the superior parietal cortex (area 5) of the rhesus monkey. Brain Res. 64, 85-102. doi: 10.1016/0006-8993(73) 90172-8

Santhanam, G., Ryu, S. I., Yu, B. M., Afshar, A., and Shenoy, K. V. (2006). A high-performance brain-computer interface. Nature 442, 195-198. doi: 10. 1038/nature04968

Scott, S. H. (2004). Optimal feedback control and the neural basis of volitional motor control. Nat. Rev. Neurosci. 5, 532-546. doi: 10.1038/ nrn 1427

Serruya, M., Hatsopoulos, N. G., Paninski, L., Fellows, M. R., and Donoghue, J. P. (2002). Brain-machine interface: instant neural control of a movement signal. Nature 416, 141-142. doi: 10.1038/416141a

Shadmehr, R., and Mussa-Ivaldi, S. (2012). Biological Learning and Control: How the Brain Builds Representations, Predicts Events and Makes Decisions. Cambridge, MA: MIT Press.

Snyder, L. H., Grieve, K. L., Brotchie, P., and Andersen, R. A. (1998). Separate body-and world-referenced representations of visual space in parietal cortex. Nature 394, 887-891. doi: 10.1038/29777 
Sommer, M. A., and Wurtz, R. H. (2008). Brain circuits for the internal monitoring of movements. Annu. Rev. Neurosci. 31, 317-338. doi: 10.1146/annurev.neuro. 31.060407.125627

Todorov, E., and Jordan, M. I. (2002). Optimal feedback control as a theory of motor coordination. Nat. Neurosci. 5, 1226-1235. doi: 10.1038/ nn963

Velliste, M., Perel, S., Spalding, M. C., Whitford, A. S., and Schwartz, A. B. (2008). Cortical control of a prosthetic arm for self-feeding. Nature 453, 1098-1101. doi: $10.1038 /$ nature 06996

Wolpert, D. M., Ghahramani, Z., and Jordan, M. I. (1995). Are arm trajectories planned in kinematic or dynamic coordinates? An adaptation study. Exp. Brain Res. 103, 460-470. doi: 10.1007/bf00241505

Zadikoff, C., and Lang, A. E. (2005). Apraxia in movement disorders. Brain 128, 1480-1497. doi: 10.1093/brain/awh560
Zago, M., McIntyre, J., Senot, P., and Lacquaniti, F. (2009). Visuo-motor coordination and internal models for object interception. Exp. Brain Res. 192, 571-604. doi: 10.1007/s00221-008-1691-3

Conflict of Interest Statement: The author declares that the research was conducted in the absence of any commercial or financial relationships that could be construed as a potential conflict of interest.

Copyright (c) 2016 Cui. This is an open-access article distributed under the terms of the Creative Commons Attribution License (CC BY). The use, distribution and reproduction in other forums is permitted, provided the original author(s) or licensor are credited and that the original publication in this journal is cited, in accordance with accepted academic practice. No use, distribution or reproduction is permitted which does not comply with these terms. 